\title{
Gol'denveizer Problem of Elastic Torus
}

\author{
Bohua $\operatorname{Sun}^{1}$ \\ ${ }^{1}$ Institute of Mechanics and Technology 8 School of Civil Engineering, \\ Xi'an University of Architecture and Technology, Xi'an 710055, China \\ http://imt.xauat.edu.cn \\ email: sunbohua@xauat.edu.cn
}

\begin{abstract}
The Gol'denveizer problem of a torus can be described as follows: a toroidal shell is loaded under axial forces and the outer and inner equators are loaded with opposite balanced forces. Gol'denveizer pointed out that the membrane theory of shells is unable to predict deformation in this problem, as it yields diverging stress near the crowns. Although the problem has been studied by Audoly and Pomeau (2002) with the membrane theory of shells, the problem is still far from resolved within the framework of bending theory of shells. In this paper, the bending theory of shells is applied to formulate the Gol'denveizer problem of a torus. To overcome the computational difficulties of the governing complex-form ordinary differential equation (ODE), the complex-form ODE is converted into a real-form ODE system. Several numerical studies are carried out and verified by finite-element analysis. Investigations reveal that the deformation and stress of an elastic torus are sensitive to the radius ratio, and the Gol'denveizer problem of a torus can only be fully understood based on the bending theory of shells.
\end{abstract}

Keywords: torus, elastic, deformation, symmetric, Gauss curvature

\section{INTRODUCTION}

Many natural and man-made objects take the shape of torus, doughnut and tube might be two of the well-known torus ( shown in Fig. 1).

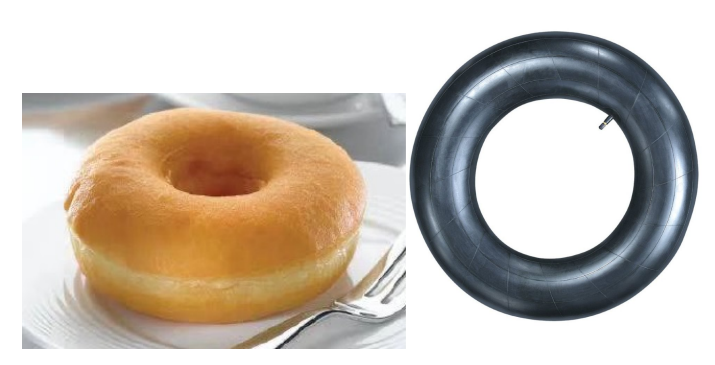

FIG. 1: Doughnut and tube.

The torus or toroidal shell, in full or partial geometric form, is widely used in structural engineering (Audoly and Pomeau 2002 [1], 2012 [2], Gol'denveizer 1961 [9], Kraus 1967 [10], Novozhilov 1959 [15], Sun 2010, 2012 [22, 23], Timoshenko and Woinowsky-Krieger 1959 [20]). Tori are interesting from an academic perspective as they are surfaces of mixed type, and little is known in general about elastic shells of mixed type (Audoly and Pomeau 2002, 2012 [1, 2], Gol'denveizer 1961 [9], Sun 2010 [22], $2012[23])$

Among regular shells, such as circular cylindrical shells, conical shells, spherical shells, and tori, the deformation of a torus is one of the most difficult topics due to its complicated topology. The source of the difficulty comes from a geometric feature of the torus, the Gauss curvature of which, $K=\frac{\sin \theta}{a(R+a \sin \theta)}$, changes its sign as the principal radius of curvature:

$$
K=\frac{1}{R a} \frac{\sin \theta}{1+\alpha \sin \theta}=\left\{\begin{array}{lc}
<0, & \theta \in[-\pi, 0] \\
=0, & \theta=0 \\
>0, & \theta \in[0, \pi]
\end{array}\right.
$$

as shown in Fig.2.

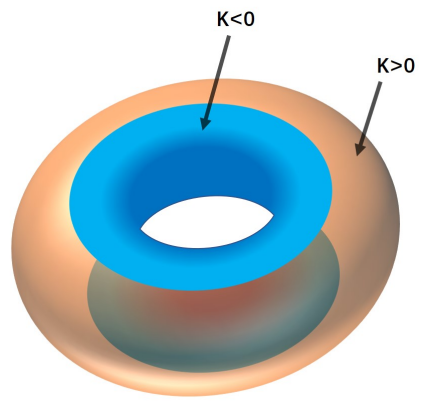

(a) Gaussian curvature signs

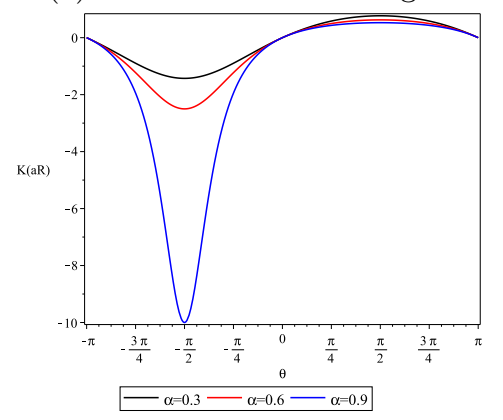

(b) Three case of the Gaussian curvature

FIG. 2: Gauss curvature $K=K_{1} K_{2}=\frac{\sin \theta}{a(R+a \sin \theta)}$, regions of positive and negative Gaussian curvature of torus of revolution.

If the principal radii are of the same sign (and, 
therefore, the Gaussian curvature of the surface $K=$ $\frac{\sin \theta}{a(R+a \sin \theta)}$ is positive), the governing equation is elliptic, and the concentrated edge loads are diffused by the shell. However, if the principal radii are of opposite sign (and, therefore, the Gaussian curvature is negative), the governing equations are hyperbolic, and at each point in the surface there are two real characteristic directions. If the characteristics run straight from edge to edge, loads applied (tangentially) at an edge are carried by undiminished tensions along these straight lines in the surface. In some respects, this makes the specification of boundary conditions awkward, since a condition applied at one edge can impose itself on a remote edge.

The torus has been studied for more than 100 years, and various aspects have been extensively investigated. Good historical reviews of a torus are given in (Audoly and Pomeau 2002, 2012 [1, 2], Chang (Zhang) 1944 [3], Clark 1950 [5], Clark and Reissner 1950 [4], Dahl 1953 [6], Flügge 1973 [7], Föppl 1907 [8], Gol'denveizer 1961 [9], Kraus 1967 [10], Kutsenko 1979 [11], Love 1902 [12], Meissner 1915 [14], Novozhilov 1959 [15], Qian and Liang 1979 [16], Reissner, E. 1949 [17], Reissner, H. 1912 [18], Tao 1959 [19], Timoshenko and Woinowsky-Krieger 1959 [20] Steele 1965 [21], Sun 2010 [22],2012 [23],2013 [24], 2018 [25], 2020 [26], Weihs 1911 [27] Wissler 1916 [28], Xia and Zhang 1986 [29], Zhang and Zhang 1991 [30], 1994 [31], Zhang, Ren and Sun 1990 [32])

Among different aspects of a torus, on page 126 of Gol'denveizer's monumental treatise on the elasticity of shells (Gol'denveizer 1961 [9]), he remarked on the membrane theory of a torus as follows. The membrane theory of shells does not yield a valid solution in the following simple situation: Imagine a toroidal thin shell loaded with surface loads that are axisymmetric with a vanishing resulting force. The inner half, with negative Gaussian curvature, feels a force $F$ directed along the axis of the torus, although the outer half, with positive Gaussian curvature, feels the opposite force $-F$ (shown in Fig.7). Membrane equations for thin shells fail to give a solution because no shear can be transmitted through the two circles with zero Gaussian curvature, i.e., the top and bottom parallels (later referred to as "extreme parallels") (Audoly and Pomeau 2002, 2012 [1, 2]).

The Gol'denveizer problem of a torus was not studied until 2002, when Audoly and Pomeau [1, 2] conducted an elegant investigation and confirmed that Gol'denveizer's remark is true. To obtain an approximated solution of torus deformation around the crowns by combining membranae solution of shell and nonlinear boundary-layer. To the best of my knowledge, the study of Audoly and Pomeau $[1,2]$ is the only research that has been done on the Gol'denveizer problem of a torus. However, the Gol'denveizer problem of a torus is still far from resolved within the framework of bending theory of shells. In light of their study (Audoly and Pomeau 2002, 2012 $[1,2])$, proposed herein is a complete solution for the Gol'denveizer problem of a torus that uses the bending theory of shells.
The rest of this paper is organized as follows. In Section 2, a formulation of the Novzhilov complex-form ordinary differential equation of a torus is presented, and the complex-ODE is split into a real-form ODE. In Section 3, the Gol'denveizer problem of a torus and the boundary conditions are presented. In Section 4, numerical simulations for three cases are carried out using custom Maple code. In Section 5, the proposed solution is verified by finite-element analysis. The functions $A(\theta), B(\theta)$, and $V(\theta)$ are studied in Section 6. Section 7 contains conclusions and future perspectives.

\section{COMPLEX-FORM FORMULATION OF SYMMETRICAL DEFORMATION OF A TORUS}

Complex-form formulation of symmetrical deformation of a torus: For the torus shown in Fig. 3, the positions of points on the middle surface will be determined by the angles $\theta$ and $\varphi$. Further, let $R_{1}$ be the radius of curvature of the meridian and $R_{2}$ the radius of curvature of the normal section, tangential to the parallel circle. This second radius is equal to the segment of the perpendicular to the middle surface between this surface and the axis of the torus.

$R$ is known as the "major radius" and $a$ as the "minor radius." The ratio $R$ divided by $a$ is known as the "aspect ratio." The typical confectionery doughnut has an aspect ratio of approximately 3 to 2 . The three classes of standard tori correspond to the three possible aspect ratios between $R$ and $a$ : When $R>a$, the surface will be the familiar ring torus or anchor ring; $R=a$ corresponds to the horn torus, which, in effect, is a torus with no "hole"; $R<a$ describes the self-intersecting spindle torus; and, when $R=0$, the torus degenerates to the sphere.

The Lamé parameters in this case are determined by the expressions $A_{1}=R_{1}=a$ and $A_{2}=R_{2} \sin \theta=R+$ $a \sin \theta$. The principal radii of curvature are given by $R_{1}=$ $a$ and $R_{2}=R \frac{1+\alpha \sin \theta}{\sin \theta}$, where $\alpha=a / R$.

The complex-form governing equation of a torus for symmetrical deformation was obtained by Novozhilov (1959) [15] as follows:

$$
(1+\alpha \sin \theta) \frac{d^{2} V}{d \theta^{2}}-\alpha \cos \theta \frac{d V}{d \theta}+2 i d^{2} \sin \theta V=P(\theta)
$$

The component of the displacement of an arbitrary point on the meridian in the direction of the axis of the torus, $\triangle_{z}(\theta)$, is

$$
\frac{d \triangle_{z}(\theta)}{d \theta}-\frac{a}{E h} \frac{R e(V(\theta)) \cos \theta}{\alpha(1+\alpha \sin \theta)}=0 .
$$

where $V(\theta)$ is a auxiliary complex function of real variable $\theta, \operatorname{Re}(\mathrm{V})$ is a real part of the function $V$, and the right-hand term is $P(\theta)=-2 d^{2}\left(2 d^{2} C+\frac{1}{2} i \alpha q a\right) \cos \theta$, in which $2 d^{2}=\frac{a^{2}}{R h} \sqrt{12\left(1-\mu^{2}\right)}, \mu$ is the Poisson ratio, $h$ 


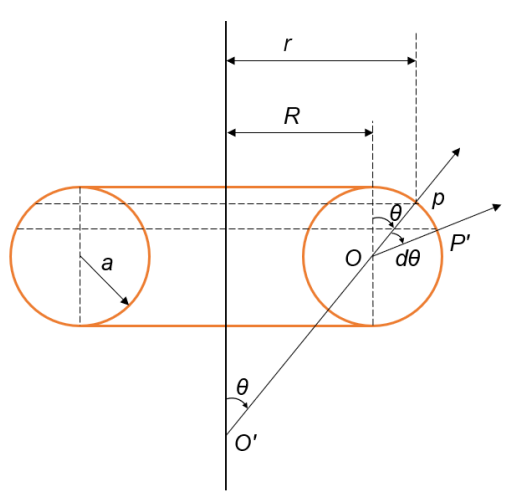

(a) Torus geometry

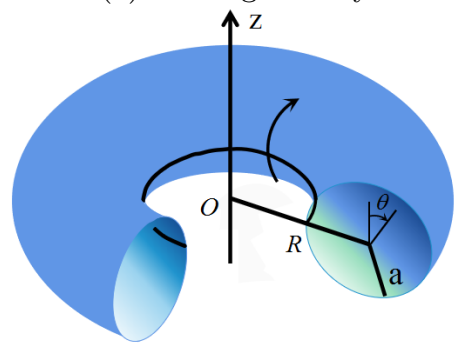

(b) Torus segment and cross-section

FIG. 3: Torus geometry: Principal radii of curvature are $R_{1}=$ $a$ and $R_{2}=a+\frac{R}{\sin \theta}$; principal curvature $K_{1}=\frac{1}{a}, K_{2}=$ $\frac{\sin \theta}{R+a \sin \theta}$; Gauss curvature $K=K_{1} K_{2}=\frac{\sin \theta}{a(R+a \sin \theta)}$.

the thickness, $q$ the distributed load, and $C$ an integration constant that can be determined from static considerations.

The analysis of the torus has thus been reduced to the problem of finding a solution for the ODEs in (2) and (3). The exact solution of Eq. 2 has been obtained and be expressed in Heun-function (Tao 1959 [19], Sun 2020 [26]), but the Heun function cannot be split into real and imaginary parts analytically. In other words, even we have the exact solution in terms of the Heun function, we do not know how to split it into its real and imaginary part that are well-known special functions. The exact solution cannot be used for computation (Sun 2020 [26]) since we need $\operatorname{Re}(\mathrm{V})$ and $\operatorname{Im}(\mathrm{V})$ to calculate the bending moments and forces in the followings.

For computational purposes, Sun (2020) citesun2020 proposed to split the function $V(\theta)$ into real and imaginary parts, i.e., $\operatorname{Re}(V)$ and $\operatorname{Im}(V)$, respectively. $V(\theta)$ is written as

$$
V(\theta)=A(\theta)+i B(\theta)
$$

with the imaginary $i=\sqrt{-1}$, where $A(\theta)=\operatorname{Re}(V)$ and $B(\theta)=\operatorname{Im}(V)$ are the real and imaginary parts of $V(\theta)$, respectively.

The bending moments $M_{1}$ and $M_{2}$ are represented by the function $A(\theta)$, and hence the function $A(\theta)$ can be called the bending function; in similar fashion, the function $B(\theta)$ can be called the membrane function since the surface forces $T_{1}$ and $T_{2}$ are expressed by it.
Substituting Eq. 4 in Eq. 2, one obtains

$$
\begin{aligned}
(1+\alpha \sin \theta) \frac{d^{2} A}{d \theta^{2}}-\alpha \cos \theta \frac{d A}{d \theta}-2 d^{2} \sin \theta B & =f(\theta), \\
(1+\alpha \sin \theta) \frac{d^{2} B}{d \theta^{2}}-\alpha \cos \theta \frac{d B}{d \theta}+2 d^{2} \sin \theta A & =g(\theta), \\
\frac{d \triangle_{z}(\theta)}{d \theta}-\frac{a}{E h} \frac{A(\theta) \cos \theta}{\alpha(1+\alpha \sin \theta)} & =0,
\end{aligned}
$$

where the free terms are $f(\theta)=-4 d^{4} C \cos \theta$ and $g(\theta)=$ $-\alpha q a d^{2} \cos \theta$.

The symmetrical deformation problem now becomes one of finding the functions $A(\theta), B(\theta)$, and $\triangle_{z}(\theta)$. The coupled differential equations (5) can be decoupled into differential equations about $A(\theta)$ and $B(\theta)$, both fourthorder ODEs, but no analytical solutions about $A(\theta)$ and $B(\theta)$ can be obtained.

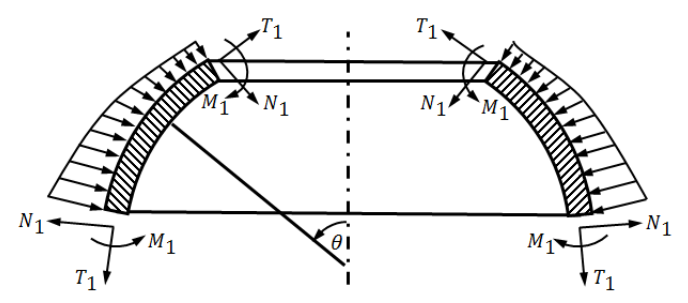

FIG. 4: Components of forces and moments on middle surface.

With these functions, one can compute all other quantities, such as $T 1, T_{2}, M_{1}, M_{2}$, and $N_{1}$, as well as $u, w$, and $\vartheta$. These quantities can be expressed in terms of $A(\theta)$ and $B(\theta)$ as follows.

The resultant moments are

$$
\begin{aligned}
& M_{1}^{*}=-\frac{\mu \alpha \cos \theta}{(1+\alpha \sin \theta)^{2}} A(\theta)-\left[\frac{d}{d \theta}\left(\frac{A(\theta)}{1+\alpha \sin \theta}\right)\right], \\
& M_{2}^{*}=-\frac{\alpha \cos \theta}{(1+\alpha \sin \theta)^{2}} A(\theta)-\mu\left[\frac{d}{d \theta}\left(\frac{A(\theta)}{1+\alpha \sin \theta}\right)\right],
\end{aligned}
$$

where the dimensionless bending moments are $M_{1}^{*}=$ $12\left(1-\mu^{2}\right) a^{2} M_{1} /\left(R h^{2}\right)$ and $M_{2}^{*}=12\left(1-\mu^{2}\right) a^{2} M_{2} /\left(R h^{2}\right)$. The resultant forces are

$$
\begin{aligned}
T_{1} & =-\frac{R h}{a^{2} \sqrt{12\left(1-\mu^{2}\right)}} \frac{\alpha \cos \theta}{1+\alpha \sin \theta} B(\theta) \\
& +\frac{q a}{2} \frac{2+\alpha \sin \theta}{1+\alpha \sin \theta}-\alpha C \frac{\alpha+\sin \theta}{(1+\alpha \sin \theta)^{2}}, \\
T_{2} & =-\frac{R h}{a^{2} \sqrt{12\left(1-\mu^{2}\right)}} \frac{d}{d \theta}\left(\frac{B(\theta)}{1+\alpha \sin \theta}\right)+\frac{q a}{2} \\
& +\alpha C \frac{\alpha+\sin \theta}{(1+\alpha \sin \theta)^{2}} .
\end{aligned}
$$

The resultant shear force is

$$
N_{1}=-\frac{h}{a \sqrt{12\left(1-\mu^{2}\right)}} \frac{\sin \theta B(\theta)-2 d^{2} C \cos \theta}{(1+\alpha \sin \theta)^{2}},
$$


and the angle of rotation of the tangent to the meridian is

$$
\vartheta=-\frac{1}{E h} \frac{A(\theta)}{\alpha(1+\alpha \sin \theta)} .
$$

The component of the displacement in the direction perpendicular to this axis is

$$
\triangle_{x}=\frac{R}{E h}(1+\alpha \sin \theta)\left(T_{2}-\mu T_{1}\right) .
$$

The bending-related shell stresses are obtained by combining the direct stresses due to the stress resultants with the flexural stresses due to the bending moments, $\sigma_{1}=\frac{T_{1}}{h} \pm \frac{6 M_{1}}{h^{2}}$, and $\sigma_{2}=\frac{T_{2}}{h} \pm \frac{6 M_{2}}{h^{2}}$.

The deformation displacement components of the middle surface in Fig. 5 can be obtained as $u=\triangle_{x} \cos \theta-$ $\triangle_{z} \sin \theta$ and $w=\triangle_{x} \sin \theta+\triangle_{z} \cos \theta$.

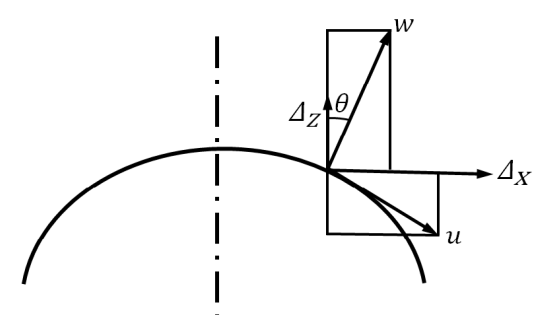

FIG. 5: $u$ and $w$ are components of displacement on middle surface.

The components of the middle-surface forces are $Q_{x}=$ $T_{1} \cos \theta+N_{1} \sin \theta$ and $Q_{z}=T_{1} \sin \theta-N_{1} \cos \theta$, where $Q_{z}$ and $Q_{x}$ are, respectively, the components of the forces in the direction of the axis of the torus and perpendicular to this axis, which act through the contemplated point of the meridian, as shown in Fig. 6.

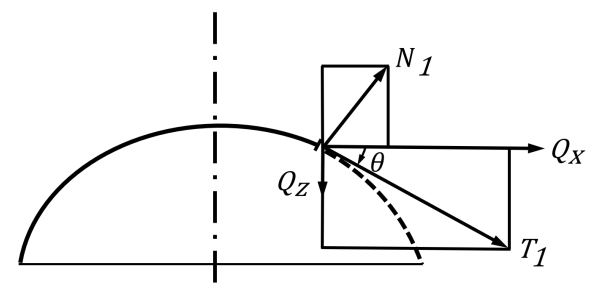

FIG. 6: $Q_{z}$ and $Q_{x}$ are components of forces on middle surface.

\section{GOL'DENVEIZER PROBLEM OF TORUS AND BOUNDARY CONDITIONS}

Gol'denveizer problem of torus and boundary conditions: The Gol'denveizer problem of torus is described as follows: a toroidal shell is loaded under axial forces shown in Fig. 7. The outer and inner equators are loaded with opposite forces $F$, so that the net force applied on the shell is balanced. Membrane theory is unable to predict deformation in this geometry, as it yields diverging stress near the crowns $\theta=0$ and $\theta=\pi$ (see Audoly and Pomeau 2002, 2012 [1, 2] for more details).

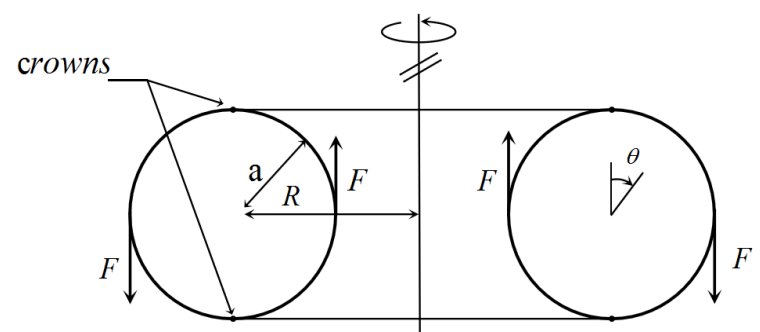

FIG. 7: Loading and geometry of Gol'denveizer problem of torus.

To obtain the correct solution, it is important to have the correct boundary conditions. First, one must identify the constant $C$. From Eq. 8, one has $T_{1}\left(-\frac{\pi}{2}\right)=$ $\frac{a}{R-a} C=\frac{F}{2 \pi(R-a)}$ and $T_{1}\left(\frac{\pi}{2}\right)=-\frac{a}{R+a} C=-\frac{F}{2 \pi(R+a)}$, both of which give a constant:

$$
C=\frac{F}{2 \pi a} \text {. }
$$

Since the torus under study is a closed and complete one, it is clear that the deformation compatibility condition, namely the deformation continuation condition, must be satisfied. In addition, the function $V(\theta)$ and all other functions must also be periodic functions of $\theta$ with period $2 \pi$, namely $V(\theta)=V(\theta+2 \pi)$.

Considering the top crown at $\theta=0$ and $\theta=2 \pi$, the deformation continuation condition, i.e., the rotation and displacement in both $\mathrm{x}$ and $\mathrm{z}$ directions, must be

$$
\begin{gathered}
\vartheta(0)=\vartheta(2 \pi), \\
\Delta_{x}(0)=\Delta_{x}(2 \pi), \\
\Delta_{z}(0)=\Delta_{z}(2 \pi) .
\end{gathered}
$$

At the crown point rotation, namely $\vartheta(0)=\vartheta(2 \pi)$, $A(0)=A(2 \pi)$; the horizontal displacement continues, $\Delta_{x}(0)=\Delta_{x}(2 \pi)$, leading to $B(0)=B(2 \pi)$ and $\frac{d B}{d \theta}(0)=$ $\frac{d B}{d \theta}(2 \pi)$.

For reference purposes, the top crown is chosen as ground zero and $\Delta_{z}(0)=0$.

Therefore, the boundary conditions of the Gol'denveizer problem of a torus are obtained as follows:

$$
\begin{aligned}
A(0) & =A(2 \pi), \\
B(0) & =B(2 \pi), \\
\frac{d B}{d \theta}(0) & =\frac{d B}{d \theta}(2 \pi), \\
\Delta_{z}(0) & =\Delta_{z}(2 \pi), \\
\Delta_{z}(0) & =0 .
\end{aligned}
$$

The problem now becomes finding $A(\theta), B(\theta)$, and $\Delta_{z}(\theta)=0$ under conditions (17). 


\section{NUMERICAL SOLUTIONS OF GOL'DENVEIZER PROBLEM OF TORUS}

Numerical solutions of Gol'denveizer problem of torus: Equations 5 and 3 are now solved for some typical cases numerically, using computational code written in Maple [13].

Unless otherwise stated, all numerical calculations in this paper are based on the data in Table I. The radius $a=0.3 k, k=1,2,3$, is varied, while other quantities are unchanged. Table I lists the geometric and material properties of the torus.

TABLE I: Data of torus $(\mathrm{k}=1,2,3)$.

\begin{tabular}{c|c|c|c|c|c|c}
\hline Quantities & $R$ & $a$ & $h$ & $E$ & $\mu$ & $F$ \\
\hline \hline Units & $\mathrm{m}$ & $\mathrm{m}$ & $\mathrm{m}$ & $N / m^{2}$ & 1 & $N$ \\
\hline Data & 1 & $0.3 k$ & 0.05 & $2.07 \times 10^{11}$ & 0.3 & 1 \\
\hline
\end{tabular}

Note: Loads are set to unit values in all simulations. Since a small deformation is a linear problem, the superposition principle can be used for different loads.

The geometry of tori with radii of $a=0.3,0.6$, and 0.9 $\mathrm{m}$ are shown in Fig. 8.

\section{A. Deformation analysis}

The horizontal and vertical displacements are shown in Figs. 9 and 10. Fig. 10 indicates that the veridical displacements are mainly contributed by the torus close to the crowns at $\theta=0$ and $\theta=\pi$; the rest of the domain is quite flat.

The numerical results of displacement components and rotation are shown in Figs. 11 and 12.

Figure11 shows that meridian displacements $u(\theta)$ are null at the top crown at $\theta=0$, and those in the inner half of the torus, $\theta \in[-\pi / 2,0]$, are larger than those in the outer half, $\theta \in[0, \pi / 2]$.

Figure12 shows that normal displacements $w(\theta)$ have four "zero" points at $\theta=0, \pi / 2, \pi$, and $3 \pi / 2$.

\section{B. Deformed cross-section of torus}

To have a better imagination on deformed torus, let's plot the deformation of torus as shown in Fig. 13:

The deformed torus in Fig. 13 is matching to the illustration proposed by Basile Audoly and Yves Pomeau 2002 [1] (as shown in Fig. 14) based on their deep insights.

\section{Bending moment and force analysis}

The numerical results of bending moment $M_{1}$ are shown in Fig. 15.

The numerical results of membrane force $T_{1}$ are shown in Fig. 16.

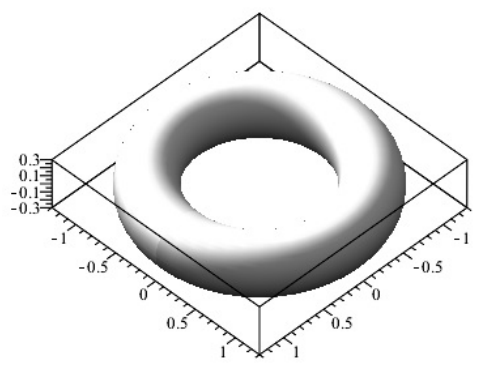

(a) $3 \mathrm{D}$ view for radius $a=0.3 \mathrm{~m}$ and $\alpha=a / R=0.3$.

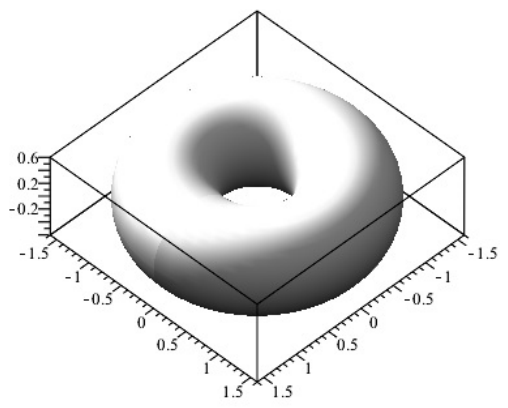

(b) $3 \mathrm{D}$ view for radius $a=0.6 \mathrm{~m}$ and $\alpha=a / R=0.6$.

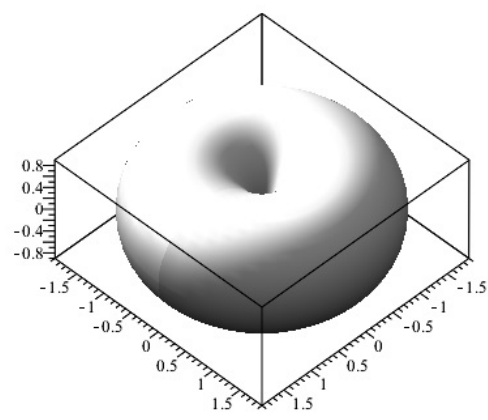

(c) $3 \mathrm{D}$ view for radius $a=0.9 \mathrm{~m}$ and $\alpha=a / R=0.9$.

FIG. 8: Three-dimensional (3D) views of torus geometries with different radii; torus in $\mathrm{c}$ is a "fat" torus.

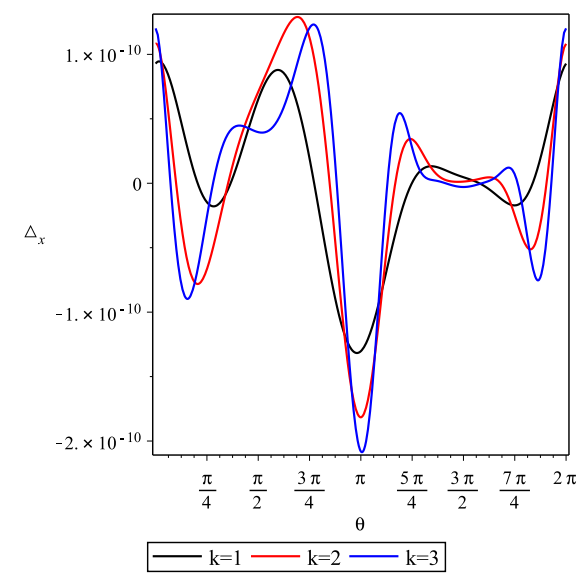

FIG. 9: Horizontal displacement $\triangle_{x}$.

The numerical results of shear forces $N_{1}$ are shown in Fig. 17. 


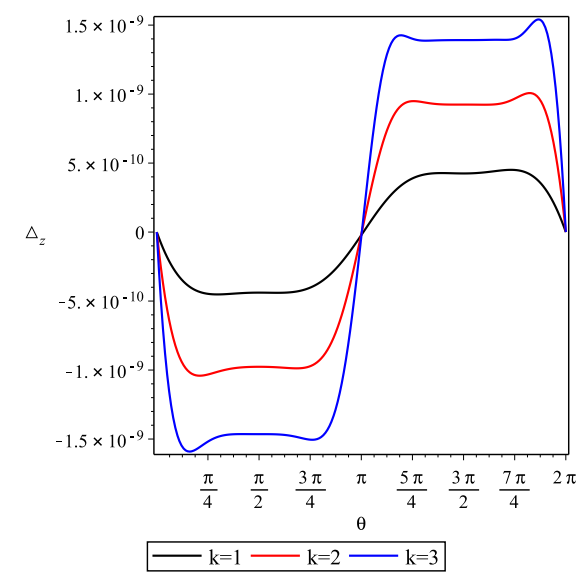

FIG. 10: Vertical displacement $\triangle_{z}$.

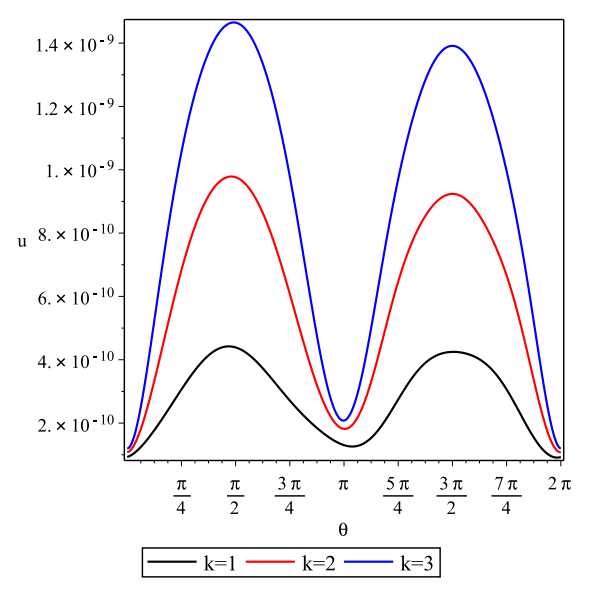

FIG. 11: Meridian displacement $u(\theta)$.

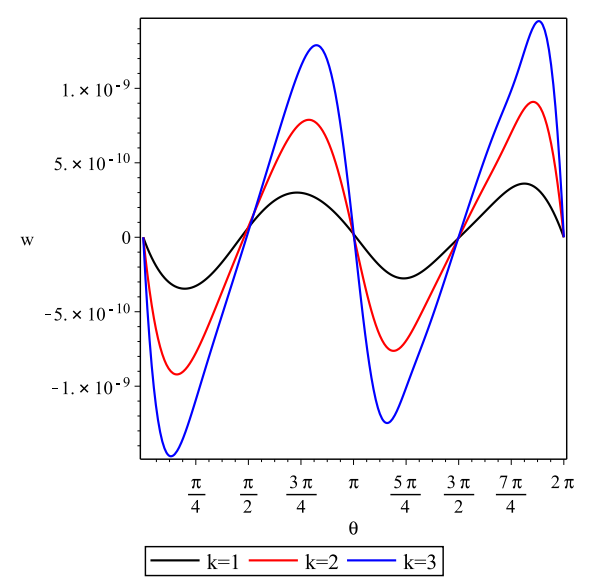

FIG. 12: Normal displacement $w(\theta)$ in domain of $\theta \in[-\pi, \pi]$.

The numerical results of stresses $\sigma_{1}$ on the surface are shown in Fig. 18.

Figures15, 16, and 17 indicate that the bending moment $M_{1}$, membrane force $T_{1}$, and shear force $N_{1}$ have a

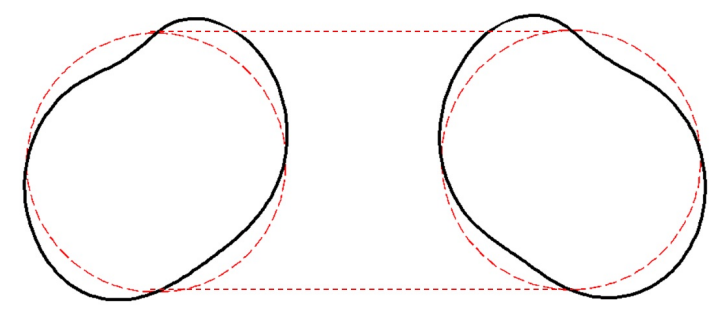

(a) Deformed shape without axes

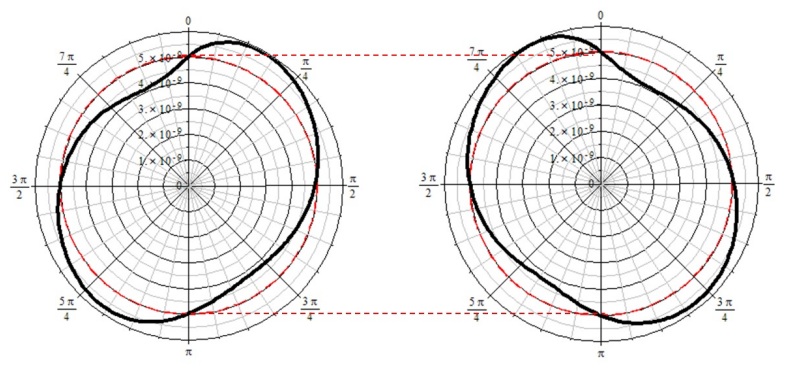

(b) Deformed shape with axes

FIG. 13: Cross-section of deformed torus for radius $a=$ $0.6[\mathrm{~m}]$. The deformed shape is plotted by using the normal displacement $w(\theta)$.

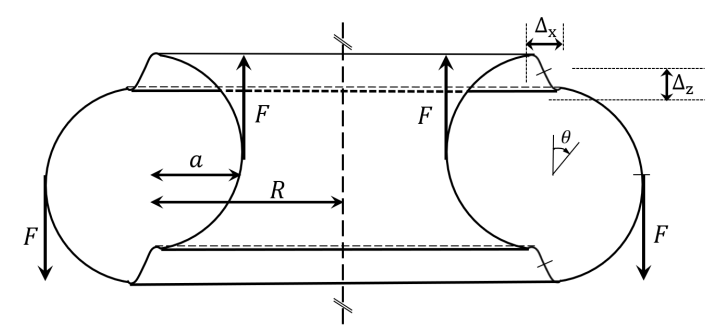

FIG. 14: Cross-section of deformed torus imagined by Basile Audoly and Yves Pomeau 2002 [1]

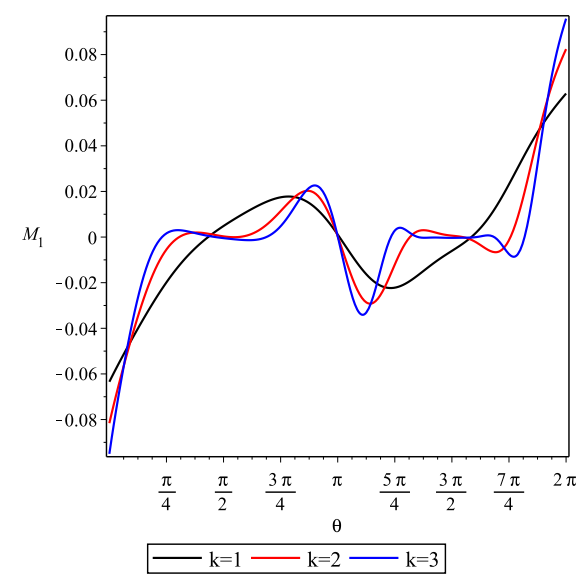

FIG. 15: Bending moment $M_{1}$. Torus radius is $a=0.3 k \mathrm{~m}$.

strong boundary-layer effect $[1,2]$ and vary dramatically with $\theta$, which clearly shows that the bending moment should not be omitted in torus analysis. 


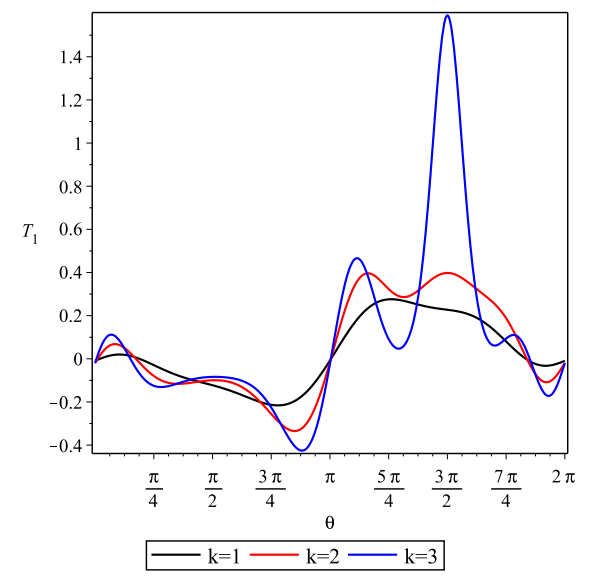

FIG. 16: Membrane force $T_{1}$. Torus radius is $a=0.3 k \mathrm{~m}$.

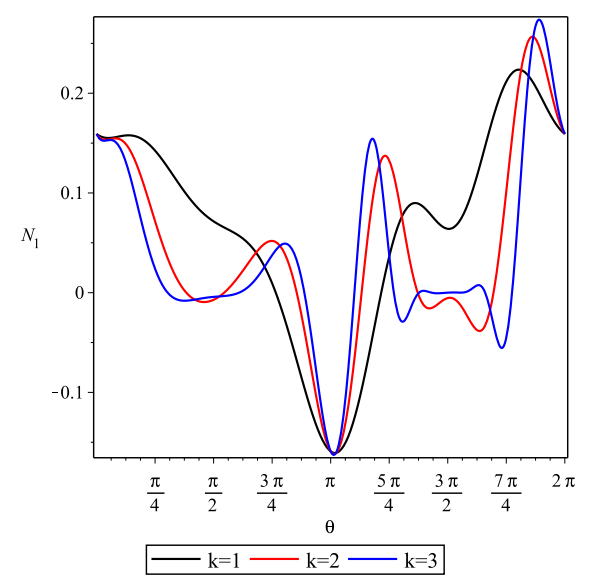

FIG. 17: Shear force $N_{1}$. Torus radius is $a=0.3 k \mathrm{~m}$.

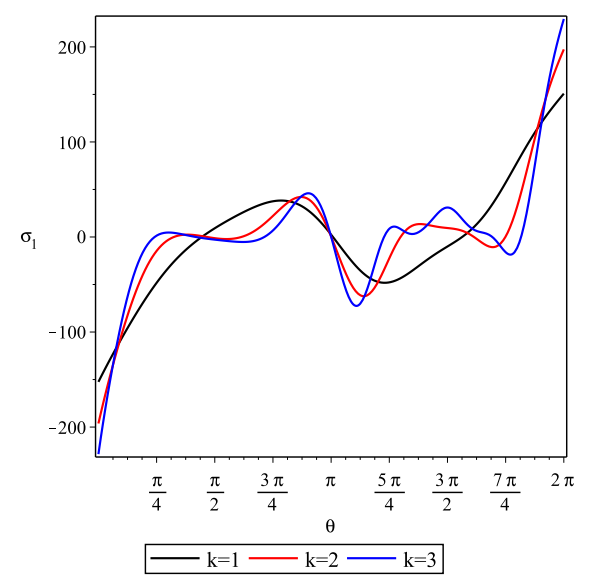

FIG. 18: Stress $\sigma_{1}$.

The above figures indicate that all quantities, such as bending moments, surface forces, shear forces, and displacements, are strongly effected by the radius ratio $\alpha=a / R$, and vary dramatically with $\theta$ both near to and far from the edge.

\section{VALIDATION OF RESULTS BY FINITE-ELEMENT ANALYSIS}

Validation of results by finite-element analysis: To verify the results obtained, finite-element analysis (FEA) was carried out for two cases.

\section{A. Torus with a cut (radius $a=0.6$ )}

The loading and geometry of this case is shown in Fig. 19.

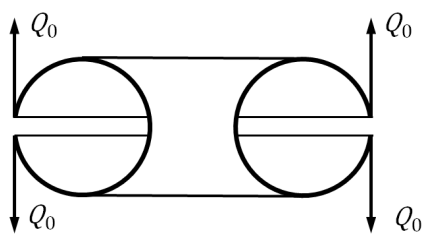

FIG. 19: Torus with a cut along its parallel at $\theta=\frac{\pi}{2}$ or $\theta=-\frac{\pi}{2}$ under load $Q_{0}=1 \mathrm{~N} / \mathrm{m}, E=2.07 \times 10^{11}\left[\mathrm{~N} / \mathrm{m}^{2}\right]$, $\mu=0.3, R=1 \mathrm{~m}, a=0.6 \mathrm{~m}$, and $h=0.05 \mathrm{~m}$.

The finite-element results were simulated by ABAQUS with shell element S4R. The boundary condition $\triangle_{z}(-3 \pi / 2)=0$ was applied to cancel the rigid body motion, which might be the reason for minor differences in the range $\theta \in[-3 \pi / 2,-5 \pi / 4]$. From a physics point of view, the result is symmetric with respect to the axes of $\theta=-\pi / 2$, while FEA reveals a slight symmetry breaking. Therefore, the results are more trustworthy since FEA supports numerical predictions nicely. Comparisons are shown in Fig. 20.

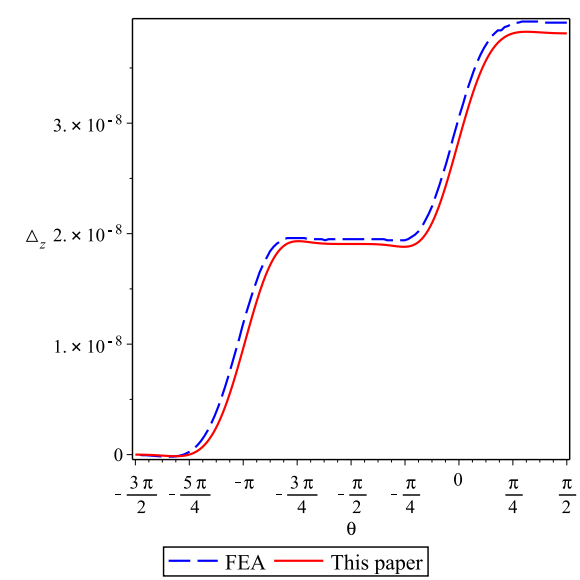

FIG. 20: Results for case shown in Fig. 19. Vertical displacement $\triangle_{z}$. Torus radius is $a=0.6 \mathrm{~m}$. 


\section{B. Goldenverizer problem (radius $a=0.6$ )}

The torus comparisons for the Goldenverizer problem are shown in Fig. 21.

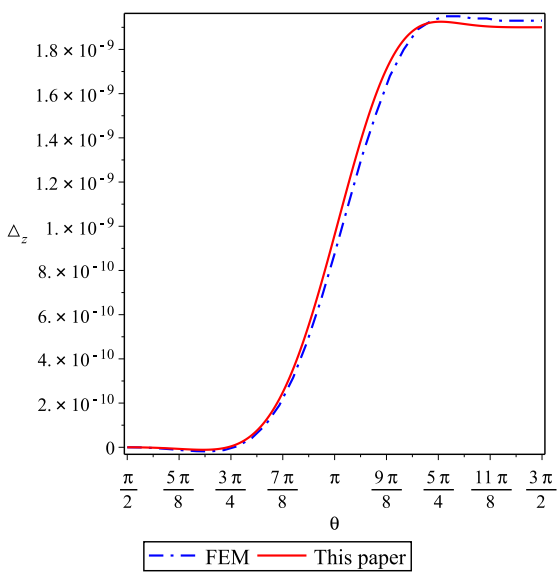

FIG. 21: Vertical displacement $\triangle_{z}$. Torus radius is $a=0.6$ m.

The finite-element results were simulated by ABAQUS with shell element S4R. The minor differences in the range $\theta \in[\pi,-3 \pi / 2]$ might come from the treatment of closed and complete tori, where the torus was cut into two parts. Nevertheless, the results are supported by the FEA results.

\section{FEATURES OF FUNCTIONS $A(\theta), B(\theta)$, AND $V(\theta)$}

Features of functions $A(\theta), B(\theta)$, and $V(\theta)$ : Owing to the importance of the functions $A(\theta), B(\theta)$, and $V(\theta)$ in the deformation analysis of the torus, it is of interest to see the features of these functions.

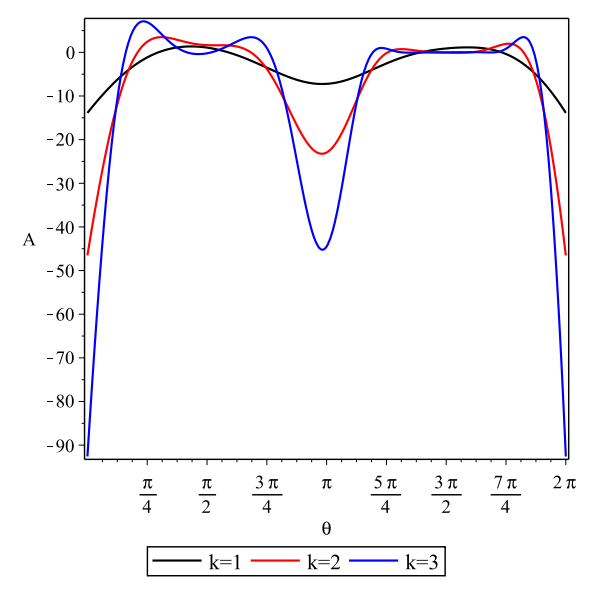

FIG. 22: Bending function $A(\theta)$.

Figure 22 shows that the membrane function $A(\theta)$ varies with the change of $\theta$.

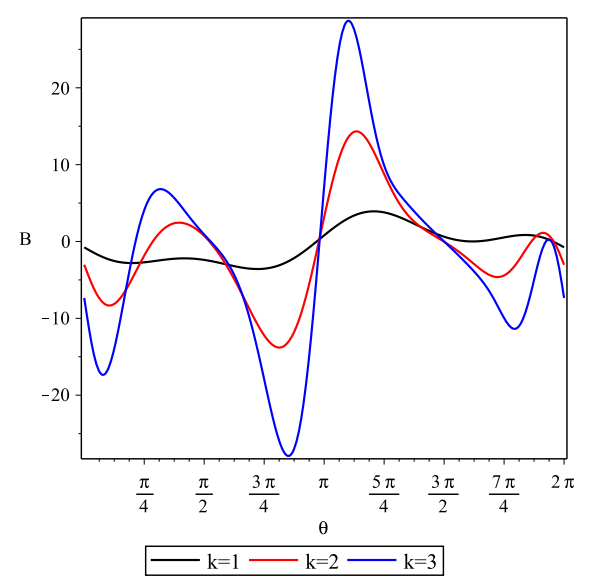

FIG. 23: Membrane function $B(\theta)$.

Figure 23 shows that the bending function $B(\theta)$ varies with the change of $\theta$.

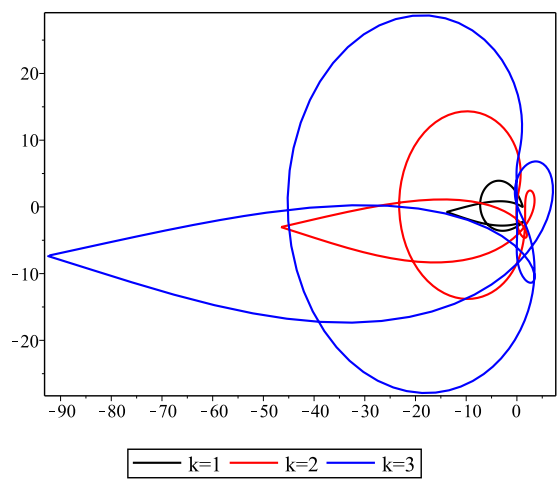

FIG. 24: Auxiliary complex function $V(\theta)=A(\theta)+\sqrt{-1} B(\theta)$.

Figure 24 is remarkable in that it shows that function $V(\theta)$ is a conformal function and its size is proportional to the ratio $\alpha=a / R$; the larger the value of alpha, the larger the size of the function $V(\theta)$ is. Inspection of the figure of $V(\theta)$ might imply some kind of deep connection between the problem and torus geometry, which should be investigated in the future.

It is worth mentioning that the figures showing the functions $A(\theta), B(\theta)$, and $V(\theta)$, to the best of my knowledge, have not been previously seen in the literature.

\section{CONCLUSIONS AND FUTURE PERSPECTIVES}

The Gol'denveizer problem of a torus is studied herein using the bending theory of a torus. To the best of my knowledge, all numerical results in this paper on the aforementioned problem have been obtained for the first time. The study confirms that the bending theory can 
provide a complete solution of the problem. To verify the presented formulation, computational code was written in Maple and several numerical simulations carried out. The validation of the numerical results was confirmed and supported by the results of finite-element analysis. The results of numerical investigation show that the bending theory of shells should be used for torus deformation analysis. The geometric properties of function $V(\theta)$ might imply a kind of deep connection with the problem, which could be one of the topics for future study. It is worth to make a note here that this problem can only be solved by using Novozhilov's formulation, rather than Reissner's and displacement formulation.

\section{Acknowledgement}

The author is honored to have benefits from my supervisor Prof. Dr.Ing Wei Zhang (Wei Chang ), who was the first Chinese scholar studied the deformation of torus, it is my privilege to dedicate this paper to the memories of Prof. Zhang for his great contribution to the analysis of elastic torus. The author appreciates my students: Mr. Guang-Kai Song, for providing finite element analysis data in Fig. 20 and 21, and preparation of Fig.4,Fig. 5 and 6; Mr. Wenqing Shao, for preparation of Fig.2(a); Mr. Zhe Liu, for drawing of Fig.3 (a); Mr. Xiang Li, for preparation of Fig.3 (b) and Fig.7; Mr. Pin-yuan Chen, for drawing of Fig. 14.

Data availability The data that support the findings of this study are available from the corresponding author upon reasonable request.

Conflict of interest The author declares that he has no known competing financial interests or personal relationships that could have appeared to influence the work reported in this paper.
[1] Audoly, B. and Pomeau, Y., 2002. The elastic torus: anomalous stiffness of shells with mixed type, C. R. Mecanique 330, 425-432.

[2] Audoly, B. and Pomeau, Y., 2010. Elasticity and Geometry - From hair curls to the non-linear response of shells. University of Cambridge, Cambridge.

[3] Chang, W. (Zhang, W.), 1944. Derspannungszustand in kreisringschale und ähnlichenSchalen mit Scheitelkreisringen unter drehsymmetrischer Belastung, Arbeitzur Erlangung des Grades eines Doctor-Ingenieurs der Technichen Hochschule, Berlin, 1944.(published in Scitific Report of Nattional Tsinghua University, Ser A. 289-349, 1949.)

[4] Clark, R.A. and Reissner, E., 1950. Bending of curved tubes, Advances in Applied Mechanics, vol. II, Academic Press.

[5] Clark, R.A., 1950. On the theory of thin elastic toroidal shells, J. Mech. Phys. Solids,29,3:146-178.

[6] Dahl, N.C., 1953. Toroidal-shell expansion joints, J. of Applied Mechanics, ASME, 20:497-503.

[7] Flügge, W., 1973. Stresses in Shells. Springer-Verlag Berlin.

[8] Föppl, L., 1907. Vorlesungen Über Technische Mechanik, volume 5. B. G. Teubner, Leipzig, Germany.

[9] Gol'denveizer, A.L., 1961. Theory of Elastic thin Shells. Pergamon Press, New York, 1961.

[10] Kraus, H., 1967. Thin Elastic Shells. John Wiley and Son, New York.

[11] Kutsenko, G. V., 1979. Axisymmetric deformation of a circular torus. International Applied Mechanics, 15(11):1049-1053.

[12] Love, A.E.H., 1902. A Treatise on the Mathematical Theory of Elasticity (3rd ed.). Cambridge University Press.

[13] Marple https://www.maplesoft.com/

[14] Meissner, E.,1915. Über und Elastizitat Festigkeit dunner Schalen, Viertelschr. D. nature.Ges., Bd.60, Zurich.

[15] Novozhilov, V.V., 1959. The Theory of Thin Shell. No- ordhoff, Groningen.

[16] Qian, W.Z. and Liang, S.C., 1979. Complex form equation and asymptotic solution. J. of Tsinghua University, 19(1): 27-47.

[17] Reissner, E., 1949. On bending of curved thin-walled tubes, Proc. National Academy of Sci., 36, 204-208.

[18] Reissner, H., 1912. Spannungen in Kugelschalen (Kuppeln). Festschrift Heinrich Müller-Breslau, A. Kröner, Leipzig, 181-193.

[19] Tao, L.N., 1959. On toroidal shells, J. of Math and Physics. 38,130-134.

[20] Timoshenko, S. and Woinowsky-Krieger, S., 1959. Theory of Plates and Shells, McGraw-Hill, New York.

[21] Steele, C.R., 1965. Toroidal pressure vessels. J. Spacecr. Rocket., 2, 937-943.

[22] Sun, B.H., 2010. Closed-form solution of axisymmetric slender elastic toroidal shells, Journal of Engineering Mechanics, 136(10),1281-1288.

[23] Sun, B.H., 2012. Toroidal Shells. Nova Novinka, New York.

[24] Sun, B.H.., 2013. Centenary studies of toroidal shells and in memory of Prof. Zhang Wei, Mechanics in Engineering, 37(3). (In Chinese)

[25] Sun, B.H, 2018. Exact solution of Qian's equation of slender toroidal shells. Mechanics in Engineering, 38(5),567569.(in Chinese)

[26] Sun, B.H., 2020. Small Symmetrical Deformation of Thin Torus with Circular Cross-Section. Preprints, 2020120420 (doi: 10.20944/preprints202012.0420.v2).

[27] Weihs, G., 1911. Über Spannungs- und Formänderungszustände in dünnen. Hohlreifen. Halle a. S.

[28] Wissler, H., 1916. Festigkeiberechung von Ringsflachen, Promotionarbeit, Zurich.

[29] Xia, Z.H. and Zhang, W., 1986. The general solution for thin-walled curved tubes with arbitrary loadings and various boundary conditions. Int. J. Pressures and Piping 26,129-144. 
[30] Zhang, R.J. and Zhang, W., 1991. Turning point solution for thin toroidal shell vibrations. Int. J. Solids Structures $27(10), 1311-1326$.

[31] Zhang, R.J. and Zhang, W., 1994. Toroidal shells under Nonsymmetric Loading, Int. J. Solids Structures,
31(19),2735-2750.

[32] Zhang, W., Ren, W.M. and Sun, B.H., 1990. Toroidal Shells - history, current situation and future (in Chinese), The 5th Conf. of Space Structures, Lanzhou, China. 